\title{
The Progenitor and Early Evolution of the Type IIb SN 2016gkg
}

\author{
L. Tartaglia ${ }^{1,2}$, M. Fraser $^{3}$, D. J. Sand ${ }^{1}$, S. Valenti ${ }^{2}$, S. J. Smartt ${ }^{4}$, C. McCully ${ }^{5,6}$, J. P. Anderson ${ }^{7}$, I. Arcavi ${ }^{5,6,18}$, N. Elias-Rosa ${ }^{8}$, \\ L. Galbany ${ }^{9}$, A. Gal-Yam ${ }^{10}$, J. B. Haislip ${ }^{11}$, G. Hosseinzadeh ${ }^{5,6}$, D. A. Howell ${ }^{5,6}$, C. Inserra ${ }^{4}$, S. W. Jha ${ }^{12}$, E. Kankare ${ }^{4}$, \\ P. Lundqvist ${ }^{13}$, K. Maguire ${ }^{4,14}$, S. Mattila ${ }^{15}$, D. Reichart ${ }^{11}$, K. W. Smith ${ }^{4}$, M. Smith ${ }^{16}$, M. Stritzinger ${ }^{17}$, M. Sullivan ${ }^{16}$, \\ F. Taddia ${ }^{13}$, and L. Tomasella ${ }^{8}$ \\ ${ }^{1}$ Texas Tech University, Physics Department, Box 41051, Lubbock, TX 79409-1051, USA; 1tartaglia@ucdavis.edu \\ ${ }^{2}$ Department of Physics, University of California, Davis, CA 95616, USA \\ ${ }^{3}$ School of Physics, O'Brien Centre for Science North, University College Dublin, Belfield, Dublin 4, Ireland \\ ${ }^{4}$ Astrophysics Research Centre, School of Mathematics and Physics, Queens University Belfast, Belfast BT7 1NN, UK \\ ${ }^{5}$ Department of Physics, University of California, Santa Barbara, CA 93106-9530, USA \\ ${ }^{6}$ Las Cumbres Observatory Global Telescope Network, 6740 Cortona Drive, Suite 102, Goleta, CA 93117, USA \\ ${ }^{7}$ European Southern Observatory, Alonso de Córdova 3107, Casilla 19, Santiago, Chile \\ ${ }^{8}$ INAF Osservatorio Astronomico di Padova, Vicolo dell'Osservatorio 5, I-35122 Padova, Italy \\ ${ }_{9}^{9}$ PITT PACC, Department of Physics and Astronomy, University of Pittsburgh, Pittsburgh, PA 15260, USA \\ ${ }^{10}$ Benoziyo Center for Astrophysics, Faculty of Physics, Weizmann Institute of Science, Rehovot 76100, Israel \\ ${ }^{11}$ University of North Carolina at Chapel Hill, Campus Box 3255, Chapel Hill, NC 27599-3255, USA \\ ${ }_{12}^{12}$ Department of Physics and Astronomy, Rutgers, the State University of New Jersey, 136 Frelinghuysen Road, Piscataway, NJ 08854, USA \\ ${ }^{13}$ Department of Astronomy and The Oskar Klein Centre, AlbaNove University Center, Stockholm University, SE-106 91 Stockholm, Sweden \\ ${ }^{14}$ European Organisation for Astronomical Research in the Southern Hemisphere (ESO), Karl-Schwarzschild-Str. 2, D-85748 Garching b. München, Germany \\ ${ }^{15}$ Tuorla Observatory, Department of Physics and Astronomy, University of Turku, Väisäläntie 20, FI-21500 Piikkiö, Finland \\ ${ }^{16}$ School of Physics and Astronomy, University of Southampton, Southampton SO17 1BJ, UK \\ ${ }^{17}$ Department of Physics and Astronomy, Aarhus University, Ny Munkegade 120, DK-8000 Aarhus C, Denmark \\ Received 2016 November 2; revised 2017 January 11; accepted 2017 January 25; published 2017 February 10
}

\begin{abstract}
We report initial observations and analysis on the Type IIb SN 2016gkg in the nearby galaxy NGC 613 . SN 2016gkg exhibited a clear double-peaked light curve during its early evolution, as evidenced by our intensive photometric follow-up campaign. SN 2016gkg shows strong similarities with other Type IIb SNe, in particular, with respect to the He I emission features observed in both the optical and near-infrared. SN 2016gkg evolved faster than the prototypical Type IIb SN 1993J, with a decline similar to that of SN 2011dh after the first peak. The analysis of archival Hubble Space Telescope images indicate a pre-explosion source at SN 2016gkg's position, suggesting a progenitor star with a $\sim$ mid-F spectral type and initial mass $15-20 M_{\odot}$, depending on the distance modulus adopted for NGC 613. Modeling the temperature evolution within 5 days of explosion, we obtain a progenitor radius of $\sim 48-124 R_{\odot}$, smaller than that obtained from the analysis of the pre-explosion images $\left(240-320 R_{\odot}\right)$.
\end{abstract}

Key words: supernovae: general - supernovae: individual (2016gkg)

Supporting material: data behind figure

\section{Introduction}

Type IIb supernovae (SNe IIb) are likely the result of the core collapse of massive stars that have lost most of their hydrogen envelope prior to explosion. At early times, and through maximum light, $\mathrm{SNe}$ IIb show hydrogen features typical of Type II SNe, which later give way to He I absorption lines similar to those observed in $\mathrm{SNe} \mathrm{Ib}$ (Filippenko et al. 1994).

Several SNe IIb caught soon after explosion exhibited "double-peaked" light curves. These have been interpreted as the signature of shock breakout cooling of a progenitor star core surrounded by extended, low-mass material (e.g., Bersten et al. 2012; Nakar \& Piro 2014). Well-studied examples of this phenomenon include SNe 1993J (e.g., Richmond et al. 1994), 2011dh (e.g., Arcavi et al. 2011; Ergon et al. 2014), 2011fu (Kumar et al. 2013), and 2013df (Morales-Garoffolo et al. 2014; Van Dyk et al. 2014). Progenitor constraints from deep pre- and post-explosion imaging (e.g., Aldering et al. 1994; Maund \& Smartt 2009 for SN 1993J) have

\footnotetext{
${ }^{18}$ Einstein Fellow.
}

revealed a picture of Type IIb's originating from yellow massive stars $\left(M_{\text {ZAMS }}=12-16 M_{\odot}\right)$ in binary systems.

Here, we present the first month of evolution of the Type IIb SN 2016gkg, progenitor constraints from pre-explosion Hubble Space Telescope (HST) imaging and its early temperature evolution. SN 2016gkg was discovered by V. Buso on 2016 September 20.19 UT, ${ }^{19} 78$ ". 4 south and 49 !. 1 west from the nucleus of NGC 613. It was confirmed via photometry (Nicholls et al. 2016; Tonry et al. 2016) and typed as a young Type II SN (Jha et al. 2016). After a fast decline (Chen et al. 2016), its light curve began to rise again toward a second maximum. Progenitor constraints from $H S T$ pre-imaging at the location of SN 2016gkg were also reported (Kilpatrick et al. 2016), and we will discuss these further below. We adopt a distance of $26 \mathrm{Mpc}$, based on a Tully-Fisher measurement ( $m-M=32.1 \pm 0.4 \mathrm{mag}$; Tully et al. 2009). However, since Tully-Fisher measurements for NGC 613 range from $\sim 20$ to $30 \mathrm{Mpc}$, we will also discuss the implications of a lower host distance $(20 \mathrm{Mpc})$. We assume

${ }^{19}$ http://ooruri.kusastro.kyoto-u.ac.jp/mailarchive/vsnet-alert/20188 

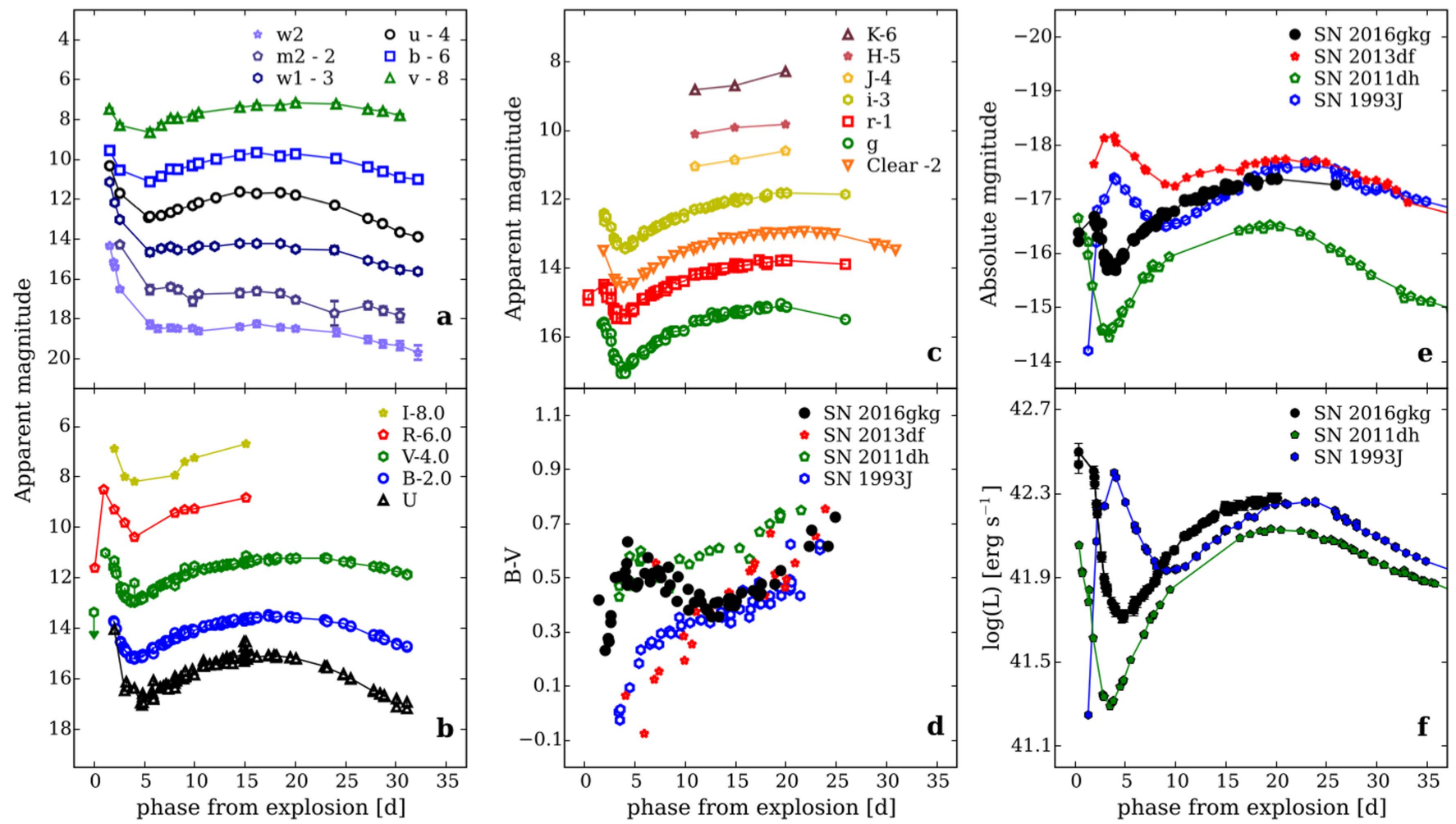

Figure 1. (a) UVOT, (b) UBV RI, and (c) and griJHK light curves of SN 2016gkg. (d) $B-V$ color evolution compared with those of SNe 1993J, 2011dh, and 2013df. (e) Absolute $r$ light curves compared with those of SNe 1993J ( $R$-band), 2011dh ( $g$-band), and 2013df ( $R$-band). (f) Pseudo-bolometric light curve compared to those of SNe 2011dh and 1993J. The pseudo-bolometric light curves were computed using Simpson's rule, integrating the photometric data. At early phase only $V r$ bands are available. For the missing bands, we assumed constant colors and a 10\% uncertainty on the computed flux. Errors for SN $2016 \mathrm{gkg}$ were computed assuming a fixed distance modulus $(\mu=32.11 \mathrm{mag})$. The data used to create this figure are available.

$A_{V}=0.053 \mathrm{mag}$ for the foreground Galactic extinction (Schlafly \& Finkbeiner 2011).

\section{Observations and Data Reduction}

\subsection{Photometry}

Imaging data were processed as follows. UBVgri frames from the Las Cumbres Observatory global telescope network (LCO; Brown et al. 2013) were reduced using LCOGTSNPIPE (e.g., Valenti et al. 2016). UBV RI data from the Public ESO Spectroscopic Survey of Transient Objects $\left(\right.$ PESSTO $\left.^{20}\right)$ using the ESO Faint Object Spectrograph and Camera v2 (EFOSC2) were reduced using the PESSTO pipeline (Smartt et al. 2015). gri data were also obtained by the Nordic Optical Telescope (NOT) Unbiased Transient Survey (NUTS ${ }^{21}$ ) with the Andalucia Faint Object Spectrograph and Camera (ALFOSC) on the NOT and reduced using a dedicated pipeline (SNOoPY; Cappellaro 2004). Filterless data from the $D<40 \mathrm{Mpc}$ (DLT40) SN search using the PROMPT5 telescope (Reichart et al. 2005) were calibrated to APASS $r$-band. ${ }^{22}$ Early Swift data (PIs: Brown, Drout) were reduced following the prescriptions of Brown et al. (2009), using the zeropoints from Breeveld et al. (2010). Data from the Asteroid Terrestrialimpact Last Alert System (ATLAS) telescope system (Tonry 2011) were also used in our early-time light curve (Arcavi et al. 2016).

\footnotetext{
${ }^{20}$ http://www.pessto.org/

21 http://csp2.1co.cl/not/

$22 \mathrm{http}$ ///www.aavso.org/apass
}

The light curves are presented in Figure 1, including publicly available early-time photometry from ASAS-SN and other sources (Nicholls et al. 2016).

\subsection{Spectroscopy}

Spectra were obtained from multiple sources, reduced in a standard manner. The classification spectrum (Jha et al. 2016) was obtained using the Southern African Large Telescope with the Robert Stobie Spectrograph and reduced using PYSALT (Crawford et al. 2010). Optical spectra from PESSTO were obtained with EFOSC2, while NIR spectra were taken with the Son OF ISAAC camera (SOFI; Moorwood et al. 1998); all of these data were reduced as in Smartt et al. (2015). FLOYDS optical spectra from LCOGT were reduced as in Valenti et al. (2014). Optical spectra from ALFOSC and the NUTS collaboration were reduced using their dedicated pipeline $\left(\right.$ FOSCGUI $^{23}$ ). Gemini south data using the NIR spectrograph FLAMINGOS-2 ( $J H$ grism, $1.0-1.8 \mu \mathrm{m}$, and $R \approx 1000$ ) were reduced using the F2 PYRAF package. The final sequence is presented in Figure 2. Spectra will be released through the Weizmann Interactive Supernova data REPository (Yaron \& Gal-Yam 2012).

\subsection{HST Pre-explosion Imaging}

NGC 613 was observed with the HST Wide-field Planetary Camera 2 (WFPC2) on 2001 August 21 (PID: 9042; PI:

$\overline{23}$ http://sngroup.oapd.inaf.it/foscgui.html 

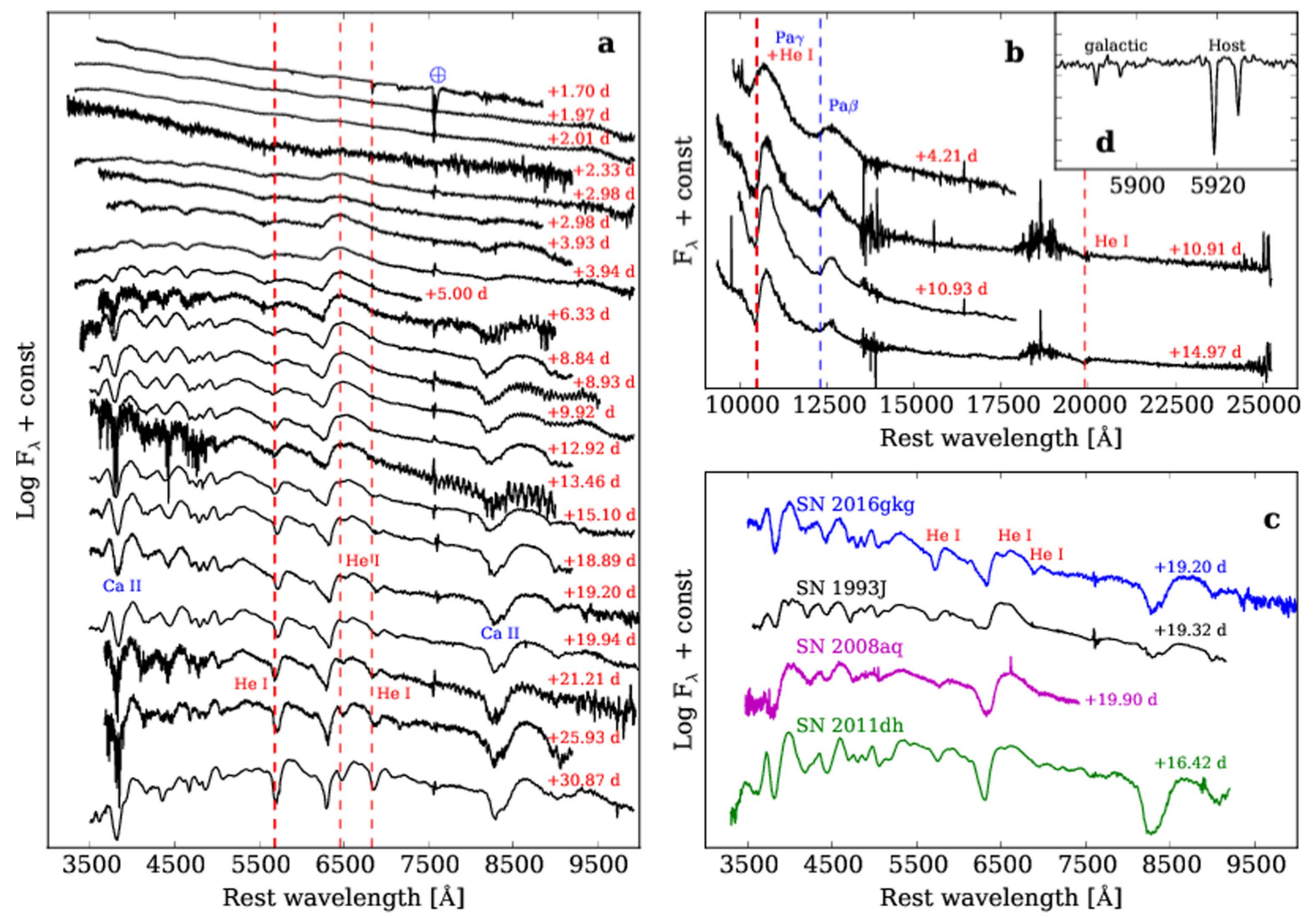

Figure 2. (a) Spectral sequence of SN 2016gkg. Helium absorption lines are marked. (b) NIR spectra. Blue and red dashed lines mark He I and H I absorption minima, respectively. (c) Comparison of SN 2016gkg with other SNe Ilb at a similar phase: SNe 1993J, 2008aq (Modjaz et al. 2014), and 2011dh. (d) High-resolution Na ID features.

S. Smartt). Exposures of $2 \times 160 \mathrm{~s}$ were taken in the $F 450 \mathrm{~W}$, $F 606 W$, and $F 814 W$ filters. The SN position falls on the WF4 chip, with a pixel scale of $0 . " 1 / \mathrm{px}$, where it lies $\sim 10$ pixels from the CCD edge.

\section{Analysis and Results}

\subsection{Light Curves}

We will adopt the discovery as the explosion epoch: $\mathrm{JD}=2457651.69028$. This is consistent with the explosion epoch obtained using the Sapir \& Waxman (2016) model to reproduce the early phase photometry (see Arcavi et al. 2016).

After an initial maximum, the light curves show a fast decline up to $\simeq+4$ days $(\mathrm{JD} \simeq 2457655)$, when the optical magnitudes increase again toward the main peak. In the Swift bands (Figure 1(a)), we note a similar decline, although the rise toward the second peak is less pronounced, with the $U V W 1$, $U V M 2$, and $U V W 2$ curves flattening after the first peak. A similar behavior was observed for SN 2011dh, but not for SN 2013df, where the UV light curves showed an almost linear decline after maximum (Morales-Garoffolo et al. 2015).

The fast early decline of SN $2016 \mathrm{gkg}$ is highlighted in Figure 1(e) where the absolute light curve is compared to those of other Type IIb SNe (SNe 1993J, 2011dh, and 2013df; Barbon et al. 1995; Arcavi et al. 2011; Morales-Garoffolo et al. 2015). The $g-$ and $B-$ band decline rates of SN $2016 \mathrm{gkg}$ $(0.58 \mathrm{mag} /$ days and $0.82 \mathrm{mag} /$ days within the first $\sim 2$ days after first maximum) are greater than those of SN 1993J in $B$ (0.31 mag/days within $\sim 5$ days), but slightly lower than those observed in SN 2011dh $(0.86 g-\mathrm{mag} /$ days within $\sim 3$ days from the first maximum).
We infer a peak absolute magnitude of $M_{B}=-16.48 \pm 0.38 \mathrm{mag}$ (where the errors in absolute magnitude here are driven by the NGC 613 distance uncertainty), rapidly decreasing to $M_{B}=-15.09 \pm 0.38$ mag within the first $\sim 2$ days of evolution. After the first peak, the absolute $B$-magnitudes are comparable with those of other $\mathrm{SNe} \mathrm{IIb}$, with an absolute magnitude for the second peak of $M_{B}=-17.03 \pm 0.38 \mathrm{mag}$.

In Figure 1(d), we compare the early $B-V$ color evolution with other Type IIb SNe. While the early evolution is similar to that observed in SN 1993J and SN 2011dh, from $\simeq+5$ days, we note an unusual flattening in the color evolution, which is not observed in other Type IIb SNe.

In Figure 1(f), we show the pseudo-bolometric light curve of SNe 2016gkg, 2011dh, and 1993J, obtained by integrating the UV through NIR fluxes. While the overall morphology of SNe 2016gkg and 2011dh is similar, the first maximum of SN 2016gkg is significantly brighter, even though their second maxima are consistent. The different decline rates implies a diversity in outer envelope extent for the three progenitors (lower in the case of SN 2011dh, larger for SN 1993J).

\subsection{Spectra}

The spectral fluxes were adjusted using photometry at similar epochs, and corrected for Galactic foreground extinction and redshift of the host $\left(v_{\odot}=1481 \pm 5 \mathrm{~km} \mathrm{~s}^{-1}\right.$; Koribalski et al. 2004). At early phases (i.e., phase $\lesssim 2.7$ days) spectra are dominated by a blue, almost featureless continuum, while P-Cygni line profiles become evident as the temperature of the ejecta decreases. 

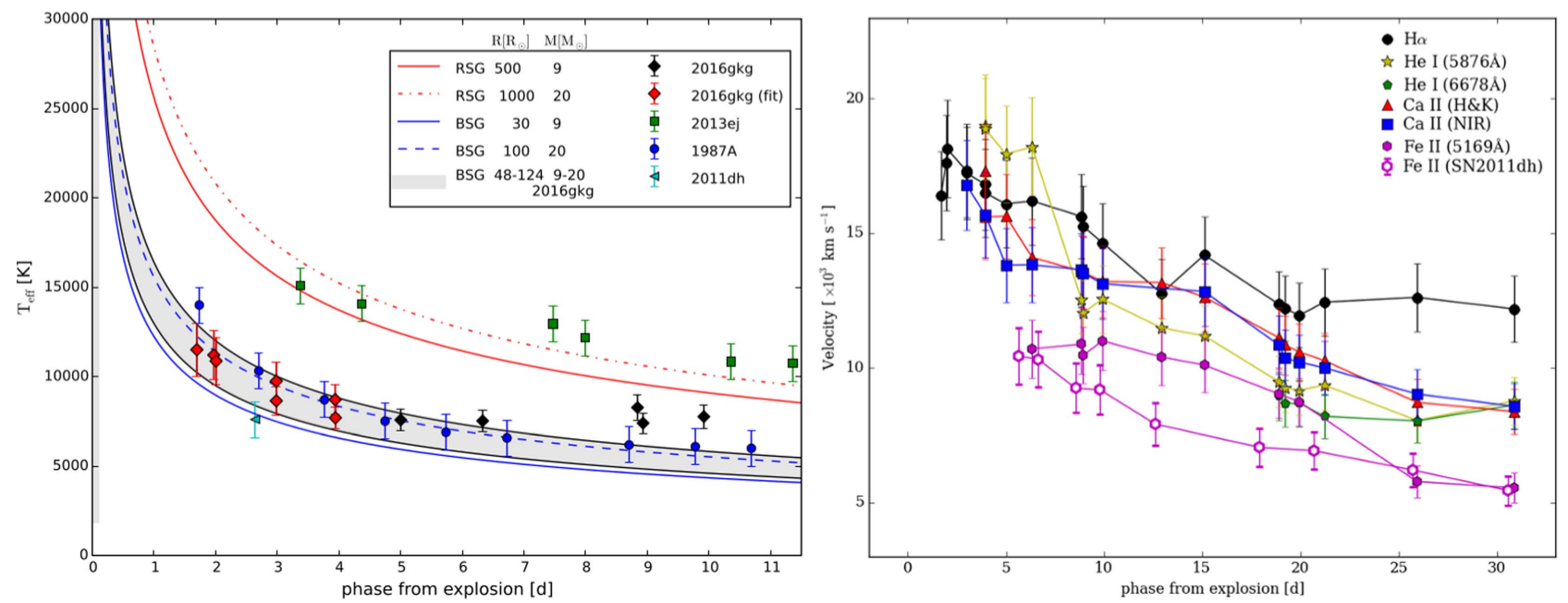

Figure 3. (a) Progenitor radius constraints using the formalism of Rabinak \& Waxman (2011) for RSG (red line), BSG (blue line), and 2016gkg (black points) and compared to those of SN 2011dh, SN 1987A (Menzies et al. 1987), and SN 2013ej (Valenti et al. 2014). The fit for SN 2016gkg was limited to +5 days from the explosion (red diamonds), following the prescriptions of Rabinak \& Waxman (2011). (b) Expansion velocity evolution for different lines. Fe II (5169 A) velocity evolution of SN 2011dh is also shown for comparison. The velocities were computed measuring the positions of the minima of the P-Cygni absorption components.

Fitting a blackbody to the spectral continuum, we infer a temperature of $\simeq 10,000 \mathrm{~K}$ at +1.70 days, rapidly decreasing to $\simeq 7000 \mathrm{~K}$ at $\sim+5$ days. We also estimated the host-galaxy reddening at the position of SN 2016gkg using the equivalent width of the Na ID doublet in an XSHOOTER spectrum $(\mathrm{EW}(\mathrm{D} 1)=0.26 \pm 0.02 \AA, \quad \mathrm{EW}(\mathrm{D} 2)=0.42 \pm 0.02 \AA$; see Figure 2(d)). Based on the correlation with the color excess (e.g., Poznanski et al. 2012), we obtain $E(B-V)_{\mathrm{NGC} 613} \lesssim$ 0.15 mag. Including this contribution, we get a slightly different temperature evolution: from $\simeq 13,000 \mathrm{~K}$ to $\simeq 7900 \mathrm{~K}$ in the first $\sim 5$ days of the spectroscopic evolution.

$\mathrm{H} \alpha$ is the most prominent line. From $\sim+14$ days a second component appears, most likely $\mathrm{He}$ I at $6678 \AA$, while $\mathrm{H} \alpha$ becomes more evident. In Figure 3(b), we report the evolution of the expansion velocities inferred from the position of the minima of the P-Cygni profile. We infer expansion velocities for $\mathrm{HI}$, declining from $\sim 16,500 \mathrm{~km} \mathrm{~s}^{-1}$ at +1.70 days to $\sim 12,200 \mathrm{~km} \mathrm{~s}^{-1}$ at $\sim+21$ days.

At $\sim+3.93$ days the $\mathrm{He} \mathrm{I}$ line at $5876 \AA$ becomes visible, with an expansion velocity decreasing from $\sim 16,500$ to $\sim 8800 \mathrm{~km} \mathrm{~s}^{-1}$ in $\sim 21$ days. The presence of helium in the early spectra of SN 2016gkg is also confirmed by our NIR spectra (Figure $2(\mathrm{~b})$ ). Our $\sim+4$ day and $\sim 11$ day spectra both show a blue continuum, with prominent Paschen lines in emission. The emission at $\sim 10750 \AA$ is most likely a blend of $\mathrm{Pa} \gamma$ and $\mathrm{He} \mathrm{I}$ at $10830 \AA$; the bluest absorption in the P-Cygni profile is consistent with the He I expansion velocity inferred from the optical spectra. From the shallow He I (20581 $\AA$ ) feature visible at $\sim+11$ days and $\sim+15$ days we infer an expansion velocity of $\sim 10,000 \mathrm{~km} \mathrm{~s}^{-1}$, in agreement with our optical spectra.

To support the classification of SN 2016gkg as a Type IIb SN, we use the Gelato (Harutyunyan et al. 2008) comparison tool on our $\sim+19$ day spectrum (see Figure 2(c)), finding good matches with Type IIb SNe (SNe 1993J, 2008aq, and 2011dh).

A detailed analysis, including the results from the complete spectroscopic follow-up campaign, will be presented in a forthcoming paper.

\subsection{Progenitor Properties}

Soon after the shock breakout, the envelope is heated by the shock, expands, and cools down. The timescale of this cooling phase depends on the initial progenitor radius, density profile, opacity, and composition.

We obtain a rough estimate on the progenitor radius of SN 2016gkg using the formalism of Rabinak \& Waxman (2011, see their Equation (12)) based on the temperature evolution within $\sim+5$ days and a typical optical opacity for H-rich material ( $\kappa=0.34 \mathrm{~cm}^{2} \mathrm{~g}^{-1}$; Rabinak \& Waxman 2011). Assuming an explosion energy of $10^{51} \mathrm{erg}$ and a mass range of $\sim 9-20 M_{\odot}$ (based on our HST data analysis; see Section 4 and Figure 5), the progenitor of SN 2016gkg is consistent with a $\sim 48-124 R_{\odot}$ star (Figure 3(a)). This radius is consistent with Kilpatrick et al. (2016), who infer a radius of $\sim 70-650 R_{\odot}$ using the Rabinak \& Waxman (2011) formalism in conjunction with the early light curves of SN $2016 \mathrm{gkg}$. We did not consider the effects of a "dark phase" of the explosion (Piro \& Nakar 2014), which would lead to an earlier explosion epoch and hence imply a larger radius for the progenitor of SN 2016gkg.

\section{The Progenitor Star}

We obtained adaptive optics imaging of SN 2016gkg with the Very Large Telescope + Nasmyth Adaptive Optics System Near-infrared Imager and Spectrograph $(\mathrm{NaCo})$ on 2016 October 2.3 UT. Ks imaging of $4500 \mathrm{~s}(75 \times 60 \mathrm{~s})$ was taken with the S54 camera, using SN 2016gkg itself as a natural guide star. Sky-subtracted images were aligned and co-added to produce a single deep post-explosion image.

Pre- and post-explosion $H S T$ and $\mathrm{NaCo}$ images were aligned using seven common reference sources. All reference sources lie on one side of the SN position, leading to some extrapolation of the geometric transformation. A transformation between the two set of pixel coordinates was derived allowing for translation, rotation, and a single magnification factor. Since so few sources were used for the alignment, we are susceptible to individual influential data points. Hence, we used a jackknife sampling technique to test the effects of excluding either one or two reference sources from the fit. We used the derived 


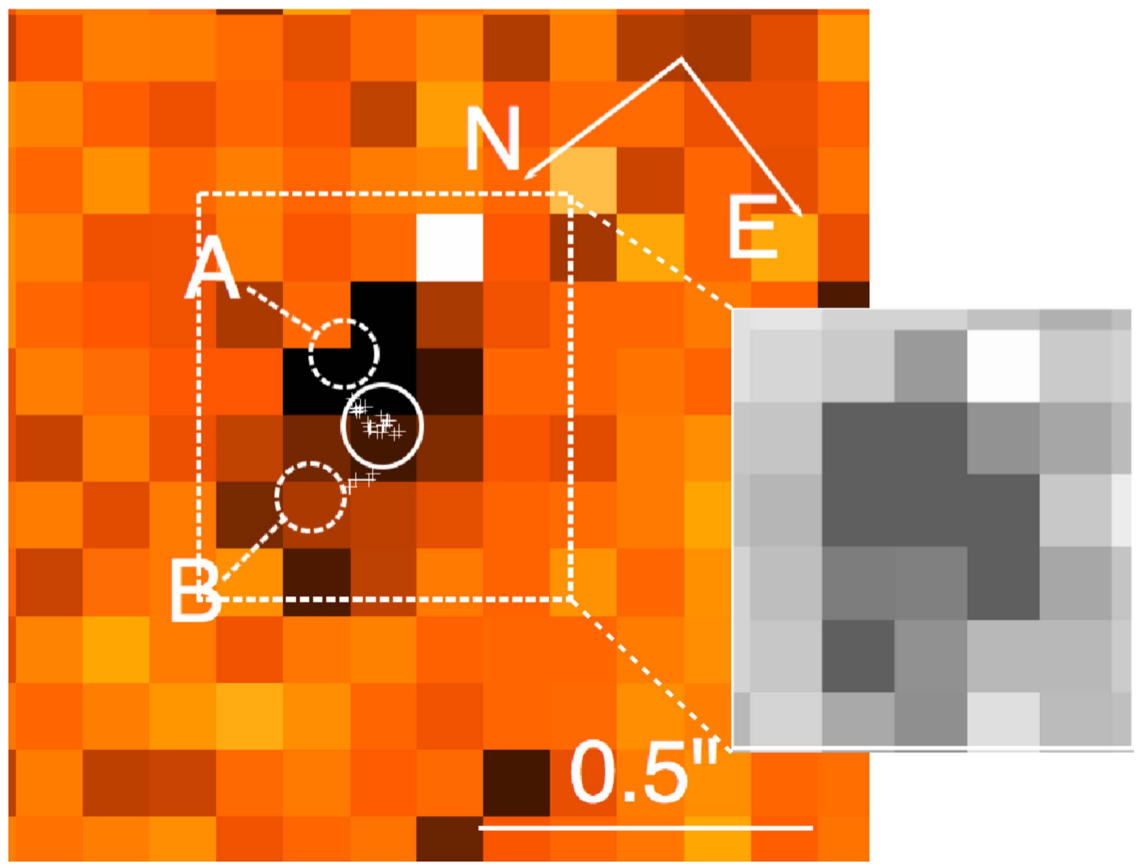

Figure 4. Region around SN 2016gkg on the pre-explosion $F 814 \mathrm{~W}$ image and a zoom-in of the region with a different contrast, more clearly showing Source B. The white pixel is a masked bad pixel. Dashed circles indicate Sources A and B: the $0 . \prime 05$ radius corresponds to their positional uncertainty. The solid ellipse is the average of the transformed, jack-knife-sampled positions (white crosses) of the $\mathrm{SN}$, with the radius corresponding to the $1 \sigma$ uncertainty (see the text for details).

transformation in each case to determine a position for the $\mathrm{SN}$ on the pre-explosion $F 814 \mathrm{~W}$ image. The resulting position lies between two sources: Source A, at pixel 586.4, 789.4 and Source B, located at 585.9, 787.3 (see Figure 4).

The positions of A and B were measured using the DOLPHOT package, while their uncertainties ( 0.5 WFPC2 pixels) were determined through Monte Carlo simulations. Source A is consistent with the $1 \sigma$ error on the transformed SN position (including the rms errors from the geometric transformations, and the uncertainties estimated from jack-knife sampling), while B lies $\sim 1.5 \sigma$ distant. Both sources are hence viable progenitor candidates for SN 2016gkg.

Both HSTPHOT and DOLPHOT (Dolphin 2000) were used for the photometry. HSTPHOT only detects Source A, with magnitudes in the VEGAMAG system $F 450 \mathrm{~W}=23.75 \pm 0.19, F 606 \mathrm{~W}=$ $23.56 \pm 0.10$, and $F 814 W=23.17 \pm 0.11 \mathrm{mag}$. The source is well fitted with a PSF, but has a sharpness parameter $(-0.31)$ that is barely consistent with point sources (between -0.3 and +0.3 ), possibly implying that Source A is slightly extended. DOLPHOT was run with standard parameters on both images in each filter simultaneously and detects both sources. We find $F 450 W=23.60 \pm 0.14, F 606 W=23.72 \pm 0.08$, and $F 814 W=23.25 \pm 0.14 \mathrm{mag}$ for $\mathrm{A}$ and $F 450 \mathrm{~W}=$ $24.52 \pm 0.37, \quad F 606 \mathrm{~W}=24.57 \pm 0.15, \quad$ and $\quad F 814 W=$ $24.13 \pm 0.29 \mathrm{mag}$ for B. Sharpness and $\chi^{2}$ are consistent with a single point source for both. For Source A, we adopt the magnitudes from HSTPhot, as this is a fully optimized tool specifically developed for WFPC 2 . We find different values for the progenitor photometry to Kilpatrick et al. (2016) (after converting their photometry from STMAG to VEGAMAG: $F 405 W=23.42, \quad F 606 W=23.10, \quad F 814 W=23.32 \mathrm{mag})$, and so we performed additional checks. The progenitor is in the Hubble Source Catalog ${ }^{24}$ with magnitude

\footnotetext{
${ }^{24}$ https://archive.stsci.edu/hst/hsc/
}

$F 450 W=23.85 \pm 0.08 \mathrm{mag}$ (converted to VEGAMAG from ABmag) and $F 606 W=23.34 \pm 0.05 \mathrm{mag}$, which is marginally brighter than the $23.56 \pm 0.10 \mathrm{mag}$ we find. We also compared the $F 606 \mathrm{~W}$ magnitudes of a set of sources on the WF4 chip in the HSC and from our own measurements with HSTPhot, and found no systematic offset between the two.

In Figure 5(a), we show the positions of A and B in a colorcolor diagram, with respect to the theoretical locus of supergiants (SGs) derived using SYNPHOT from ATLAS9 models (Castelli \& Kurucz 2004): while their colors are slightly offset from the models, they are broadly consistent with a yellow or blue supergiant.

We did not correct the magnitudes for foreground Galactic ( $A_{V}=0.05 \mathrm{mag}$ ) or host extinction, since it is small compared to the uncertainty on distance, and will have minimal effect on the bolometric correction (see also Figure 5(a)). In addition, we cannot exclude the presence of significant circumstellar dust around the progenitor, which would then be destroyed during the shock breakout (e.g., Fraser et al. 2012).

The large uncertainty on the distance of NGC 613 plays an important role in the identification of a plausible progenitor for SN 2016gkg. A variety of distances are in the literature, spanning from $\sim 19.6$ to $\sim 31.7 \mathrm{Mpc}(\mu=31.46 \pm 0.80$ and $\mu=32.51 \pm 0.47 \mathrm{mag}$, respectively). In Figure 5(b), we show the position of $\mathrm{A}$ in the Hertzsprung-Russell diagram with respect to a set of evolutionary tracks computed with the Cambridge STARS models (Eldridge \& Tout 2004). Adopting a distance modulus $\mu=32.11 \pm 0.38 \mathrm{mag}$, a solar metallicity, the recommended values of temperature and surface gravity for F5I stars $\quad\left(T_{\text {eff }}=6900 \mathrm{~K}\right.$ and $\log g=+1.44$ dex; Schmidt-Kaler 1982), and assuming a 0 mag bolometric correction (see, e.g., Girardi et al. 2008), we obtain $M_{\mathrm{bol}}=-8.55 \pm 0.40$, mag. Although A and B sit close to the $26 M_{\odot}$ and $15 M_{\odot}$ tracks, respectively, none of them is a plausible SN progenitor, since they correspond to stars during 

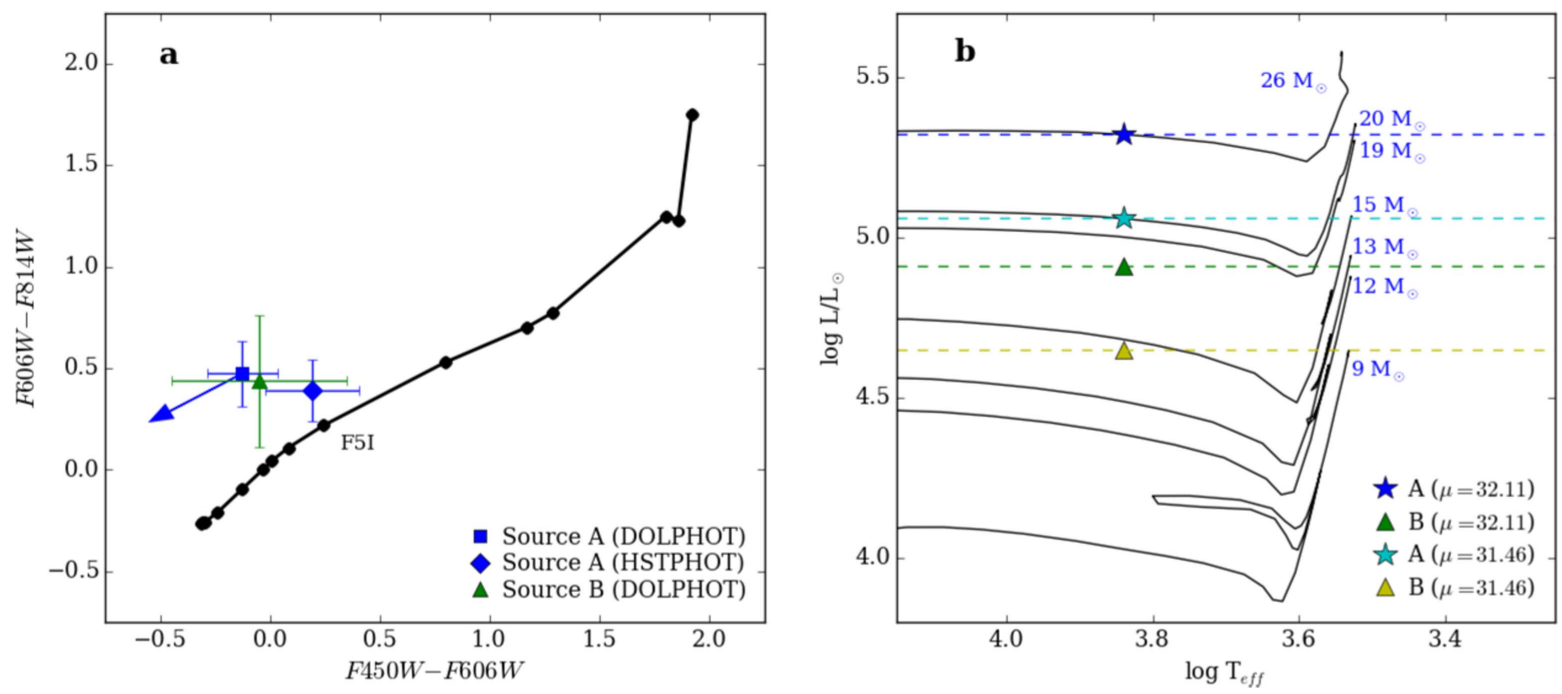

Figure 5. (a) Color-color diagram showing the positions of Sources A and B with respect to the theoretical locus of SG stars obtained using ATLAS9 models with solar metallicities. A reddening vector corresponding to an extinction of $A_{V}=1 \mathrm{mag}$ is also shown. (b) Hertzsprung-Russell diagram showing the positions of A and B with respect to evolutionary tracks computed using the STARS models, assuming solar metallicity. We also show the effects of the large uncertainties on the distance modulus (see the text for details).

the He core burning phase (see Smartt et al. 2009). Alternatively, the yellow color could be explained by additional stripping experienced by the progenitor from a stellar companion (see also Kilpatrick et al. 2016).

Hence, we compare their luminosities with the endpoint of the evolutionary tracks (dashed lines in Figure 5(b)). Source A has $\log L / L_{\odot}=5.32 \mathrm{dex}$, corresponding to the $19-20 M_{\odot}$ tracks, while $\mathrm{B}$ falls in the region where the $12-13 M_{\odot}$ tracks end, with $\log L / L_{\odot}=4.91$ dex. These luminosity and temperature estimates give radii of $\sim 320 R_{\odot}$ and $200 R_{\odot}$ for $\mathrm{A}$ and $\mathrm{B}$, respectively. If $\mathrm{A}$ is the progenitor star of SN $2016 \mathrm{gkg}$, this would imply a yellow SG more luminous than the progenitor of SN 1987A (White \& Malin 1987; Walborn et al. 1989). On the other hand, adopting $\mu=31.46 \pm 0.80(D=19.6 \mathrm{Mpc})$ for NGC 613, we obtain $M_{\mathrm{bol}, A}=-7.90 \pm 0.81 \mathrm{mag}$ and $M_{\mathrm{bol}, B}=-6.89 \pm$ $0.81 \mathrm{mag}, \quad$ corresponding to $\log L_{A} / L_{\odot} \simeq 5.06 \mathrm{dex}$ and $\log L_{B} / L_{\odot} \simeq 4.65$ dex. This would imply lower masses and radii for both Sources $\mathrm{A}$ and $\mathrm{B}\left(\sim 15 M_{\odot}\right.$, with $R \simeq 240 R_{\odot}$ and $\sim 9 M_{\odot}$, with $R \simeq 150 R_{\odot}$, respectively) This analysis suggests a larger radius for the progenitor of SN 2016gkg, arguing against the predictions of the Rabinak \& Waxman (2011) model.

Given the large uncertainties, we have no arguments to favor a particular distance for NGC 613, and we cannot exclude any of the mentioned values of the mass for the candidate progenitor. Also, given the SN position in the HST images, we lack the astrometric accuracy to exclude either of the two sources as a progenitor for SN 2016gkg, rule out the presence of a stellar companion (see Kilpatrick et al. 2016) or even exclude the possibility that the sources we have discussed are in fact star clusters. An improved distance to NGC 613 will address the first issue, while late-time imaging will clarify direct progenitor constraints.

\section{Summary and Conclusions}

The spectra of SN $2016 \mathrm{gkg}$ closely resemble those of other Type IIb SNe, in particular SNe 1993J and 2011dh. He I lines, the classical features for this class of transients, are visible since +8.82 days, becoming stronger with time.

Our photometric analysis reveals double-peaked light curves in all optical and UV bands, with a relatively fast decline in $g$ and $B$ - bands, faster than in SN 1993J, but comparable to SN 2011dh.

Deep HST pre-explosion images, and the temperature evolution within the first $\sim 5$ days from explosion, help to constrain the physical properties of the progenitor. Following Rabinak \& Waxman (2011), we obtain a radius consistent with a $\sim 48-124 R_{\odot}$ supergiant. The analysis of the archival HST images revealed the presence of two sources at the position of SN 2016gkg (Sources A and B). Source A seems to correspond to a yellow supergiant star with an initial mass and radius in the range $15-20 M_{\odot}$ and $240-320 R_{\odot}$, while $\mathrm{B}$ corresponds to a 9-13 $M_{\odot}$ star with $R \simeq 150-200 R_{\odot}$. These radii are larger than those obtained adopting the Rabinak \& Waxman (2011) formalism. Like in SN 2011dh, this might be due to the progenitor structure required to produce the double-peaked light curve observed in Type IIb $\mathrm{SNe}$ (i.e., the presence of an extended, $\quad R \simeq 10^{13} \mathrm{~cm}$, low-mass, $\quad M \leqslant 0.1 M_{\odot}$ envelope becoming transparent after a few days of expansion; see Bersten et al. 2012; Nakar \& Piro 2014).

Given the large uncertainties on the distance of NGC 613, and the unfavorable position in the HST images, we cannot rule out either of the two sources as the possible progenitor star, as well as the presence of a stellar companion. Deep late-time post-explosion HST images centered on the position of SN $2016 \mathrm{gkg}$ will provide the final word on the nature of the progenitor system.

Based on data from: ESO as part of PESSTO (197.D1075.191.D-0935) and under programme 097.D-0762(A). Gemini Observatory under GS-2016B-Q-22 (PI: Sand). The Nordic Optical Telescope of the Instituto de Astrofísica de Canarias. The European Organisation for Astronomical Research in the southern hemisphere under ESO programme 198.A-0915(A). FOSCGUI is a graphic user interface aimed at extracting SN spectroscopy and photometry obtained with FOSC-like instruments, developed by E. Cappellaro. D.J.S. 
acknowledges NSF grants AST-1412504 and AST-1517649. S.J.S. acknowledges EU/FP7-ERC grant 29122. M.F. acknowledges the support of a Royal Society-Science Foundation Ireland University Research Fellowship. L.G. was supported in part by the US National Science Foundation under grant AST-1311862. M.S. acknowledges support from EU/ FP7-ERC grant number 615929. K.M. acknowledges support from STFC through an Ernest Rutherford Fellowship. D.A.H., C.M., and G.H. are funded by NSF AST-1313484. Support for I.A. was provided by NASA through the Einstein Fellowship Program, grant PF6-170148. N.E.R. acknowledges financial support by the 1994 PRIN-INAF 2014 (project "Transient universe: unveiling new types of stellar explosions with PESSTO") and by MIUR PRIN 2010-2011, "The dark universe and the cosmic evolution of baryons: from current surveys to Euclid." M.S. acknowledges support from the Danish Agency for Science and Technology and Innovation via a Sapere Aude Level 2 grant, the Villum foundation, and the Instrumentcenter for Danish Astrophysics (IDA).

\section{References}

Aldering, G., Humphreys, R. M., \& Richmond, M. 1994, AJ, 107, 662 Arcavi, I., Gal-Yam, A., Yaron, O., et al. 2011, ApJL, 742, L18

Arcavi, I., Hosseinzadeh, G., Brown, P. J., et al. 2016, ApJL, in press (arXiv:1611.06451)

Barbon, R., Benetti, S., Cappellaro, E., et al. 1995, A\&AS, 110, 513

Bersten, M. C., Benvenuto, O. G., Nomoto, K., et al. 2012, ApJ, 757, 31

Breeveld, A. A., Curran, P. A., Hoversten, E. A., et al. 2010, MNRAS, 406, 1687

Brown, P. J., Holland, S. T., Immler, S., et al. 2009, AJ, 137, 4517

Brown, T. M., Baliber, N., Bianco, F. B., et al. 2013, PASP, 125, 1031

Cappellaro, E. 2004, SNOoPy: a package for SN photometry, http://graspa. oapd.inaf.it/snoopy.html

Castelli, F., \& Kurucz, R. L. 2004, arXiv:astro-ph/0405087

Chen, P., Dong, S., Bose, S., et al. 2016, ATel, 9529

Crawford, S. M., Still, M., Schellart, P., et al. 2010, Proc. SPIE, 7737, 773725

Dolphin, A. E. 2000, PASP, 112, 1383
Eldridge, J. J., \& Tout, C. A. 2004, MNRAS, 353, 87

Ergon, M., Sollerman, J., Fraser, M., et al. 2014, A\&A, 562, A17

Filippenko, A. V., Matheson, T., \& Barth, A. J. 1994, AJ, 108, 2220

Fraser, M., Maund, J. R., Smartt, S. J., et al. 2012, ApJL, 759, L13

Girardi, L., Dalcanton, J., Williams, B., et al. 2008, PASP, 120, 583

Harutyunyan, A. H., Pfahler, P., Pastorello, A., et al. 2008, A\&A, 488, 383

Jha, S. W., Van Wyk, V., \& Vaisanen, P. 2016, ATel, 9528

Kilpatrick, C. D., Foley, R. J., Abramson, L. E., et al. 2016, MNRAS, 465, 4650

Koribalski, B. S., Staveley-Smith, L., Kilborn, V. A., et al. 2004, AJ, 128, 16

Kumar, B., Pandey, S. B., Sahu, D. K., et al. 2013, MNRAS, 431, 308

Maund, J. R., \& Smartt, S. J. 2009, Sci, 324, 486

Menzies, J. W., Catchpole, R. M., van Vuuren, G., et al. 1987, MNRAS, 227, 39P

Modjaz, M., Blondin, S., Kirshner, R. P., et al. 2014, AJ, 147, 99

Moorwood, A., Cuby, J.-G., \& Lidman, C. 1998, Msngr, 91, 9

Morales-Garoffolo, A., Elias-Rosa, N., Benetti, S., et al. 2014, MNRAS, 445,1647

Morales-Garoffolo, A., Elias-Rosa, N., Bersten, M., et al. 2015, MNRAS, 454, 95

Nakar, E., \& Piro, A. L. 2014, ApJ, 788, 193

Nicholls, B., Brown, J. S., Dong, S., et al. 2016, ATel, 9521

Piro, A. L., \& Nakar, E. 2014, ApJ, 784, 85

Poznanski, D., Prochaska, J. X., \& Bloom, J. S. 2012, MNRAS, 426, 1465

Rabinak, I., \& Waxman, E. 2011, ApJ, 728, 63

Reichart, D., Nysewander, M., Moran, J., et al. 2005, NCimC, 28, 767

Richmond, M. W., Treffers, R. R., Filippenko, A. V., et al. 1994, AJ, 107, 1022

Sapir, N., \& Waxman, E. 2016, arXiv:1607.03700

Schlafly, E. F., \& Finkbeiner, D. P. 2011, ApJ, 737, 103

Schmidt-Kaler, T. 1982, 4.1.1 Classification of Stellar Spectra: Datasheet from Landolt-BÖrnstein, Vol. 2B: Stars and Star Clusters (Berlin: Springer)

Smartt, S. J., Eldridge, J. J., Crockett, R. M., \& Maund, J. R. 2009, MNRAS, 395, 1409

Smartt, S. J., Valenti, S., Fraser, M., et al. 2015, A\&A, 579, A40

Tonry, J., Denneau, L., Stalder, B., et al. 2016, ATel, 9526

Tonry, J. L. 2011, PASP, 123, 58

Tully, R. B., Rizzi, L., Shaya, E. J., et al. 2009, AJ, 138, 323

Valenti, S., Howell, D. A., Stritzinger, M. D., et al. 2016, MNRAS, 459, 3939

Valenti, S., Sand, D., Pastorello, A., et al. 2014, MNRAS, 438, L101

Van Dyk, S. D., Zheng, W., Fox, O. D., et al. 2014, AJ, 147, 37

Walborn, N. R., Prevot, M. L., Prevot, L., et al. 1989, A\&A, 219, 229

White, G. L., \& Malin, D. F. 1987, Natur, 327, 36

Yaron, O., \& Gal-Yam, A. 2012, PASP, 124, 668 